\title{
\&UIIBRAGEC \\ REPRESENTAÇÕES GRÁFICAS PARA UMA VISÃO CONJUNTA DE LEAN CONSTRUCTION E LINHA DE BALANÇO ${ }^{1}$
}

\author{
GOMES, Maria Micheline Barreto (1); OLIVEIRA, Marisônia Benevides Pinheiro \\ de (2); HEINECK, Luiz Fernando Mählmann (3) \\ (1) Universidade Federal do Ceará, linebg_ce@yahoo.com.br (2) Universidade Federal do Ceará, \\ marisbenevides@yahoo.com.br (3) Universidade Estadual do Ceará, luiz.heineck@uece.br
}

\begin{abstract}
RESUMO
O processo de planejamento e controle da produção exerce um papel fundamental nas empresas, à medida que o mesmo tem um forte impacto no desempenho da função produção. Diversas técnicas de planejamento e controle de projetos são utilizadas, sendo a Linha de Balanço a mais indicada para projetos com serviços repetitivos, uma vez que utiliza conceitos de fluxo de produção, repetição de ciclos e estabelece a coordenação entre esses fluxos de produção. Dessa forma, esse estudo teve por objetivo uma exploração gráfica sobre os conceitos da Lean Construction e sua ligação com a Linha de Balanço. Para isso realizou-se um estudo teórico feito através de revisão de literatura sobre o assunto e um levantamento de gráficos que relacionam termos da Lean Construction com essa técnica de programação. Como resultado, obteve-se uma ampla apresentação gráfica sobre os ciclos e os fluxos na construção civil e como os mesmos ocorrem ao longo do tempo, além de verificar a semelhança desses com a Linha de Balanço. Conclui-se por meio destas representações, que os conceitos da Lean Construction e a da Linha de Balanço podem ser trabalhados em conjunto.
\end{abstract}

Palavras-chave: Linha de balanço, Lean Construction, Ensino de Engenharia.

\begin{abstract}
The production planning and control process plays a fundamental role in building companies, as it has a strong impact on the performance of the production function. Several project planning and control techniques are used, the Line of Balance Line being the most suitable for projects with repetitive services, since it uses concepts of production flow, repetition of cycles and establishes coordination between such production flows. Thus, this study aimed at a graphic exploration of the Lean Construction concepts and their connection with the Line of Balance.. For this, a theoretical study was carried out through a literature review on the subject and a graphic survey relating the terms of Lean Construction to this programming technique. As a result, a wide graphic presentation was obtained on cycles and flows in building construction and how they occur over time. It is concluded through the graphical representations, that the concepts of Lean Construction and Line of Balance can be analysed in a unique academic setting.
\end{abstract}

Keywords: Line of balance, Lean construction, Engineering Education.

\section{INTRODUÇÃO}

A área de PCP tem sido dominada, em anos recentes pelos trabalhos relativos à LBMS (Locational Based Management System) e seus correlatos como Takt Planning e Determinação de Pulmões de atividades (os autores, Seppänen e Tommelein são exemplos disto na lista bibliográfica), podem ser remetidos às contribuições originais relativas à Linha de Balanço como descritas em Lumsden (1968) e apresentadas para o

${ }^{1}$ GOMES, M. M. B.; OLIVEIRA, M. B. P. de; HEINECK, L. F. M. Representações gráficas para uma visão conjunta de lean construction e linha de balanço. In: SIMPÓSIO BRASILEIRO DE GESTÃO E ECONOMIA DA CONSTRUÇÃO, 12., 2021, Maceió. Anais[...] Porto Alegre: ANTAC, 2021. p.1-8. Disponível em: https://eventos.antac.org.br/index.php/sibragec/article/view/549. Acesso em: 2 out. 2021. 
cenário brasileiro por Maders (1987). Já em 1991, Formoso incorpora os conceitos básicos da técnica em sistemas computacionais capazes de delinear, por si só, os parâmetros básicos desta técnica de programação e sua representação.

Objetiva-se assim, a partir do presente trabalho, dar a entender que a técnica de linha de balanço é capaz de ilustrar um conjunto amplo das considerações relativas ao PCP, como evidenciado pelas publicações dos últimos 30 anos. A técnica seria o repositório do conhecimento desenvolvido na área, ao se mostrar robusta em seus parâmetros para acomodar o que vem sendo apresentado na literatura sobre planejamento de obras.

Este artigo utiliza-se em grande parte do que é apresentado em um trabalho de conclusão de curso (PINHEIRO, 2009) que apresenta cerca de 70 figuras ilustrando a linha de balanço como o veículo de apresentação de 20 conceitos do que se entende por Produção Enxuta aplicada a canteiros de obras repetitivos. Estes elementos foram retirados de um Curso a nível de Pós-graduação, dividido em 10 secções, com cerca de 320 lâminas, assemelhadas ao que será apresentado de forma condensada em 30 desenhos.

Em específico, neste trabalho é apresentado o material relativo a fluxos, um dos pontos basilares da Construção Enxuta. Em artigos que se seguem, são apresentadas as demais ligações da representação gráfica em linha de balanço com outras áreas, ações e estratégias gerenciais para canteiro de obras, como consumo de recursos e uso de folgas.

\section{REVISÃO BIBLIOGRÁFICA}

A representação gráfica do progresso em obra utilizando conceitos de linha de balanço, dentro da comunidade de pesquisa do International Group for Lean Construction é iniciada com os trabalhos pioneiros de Howell e Ballard nos congressos iniciais dos anos de 1993 a 1995, reunidos em uma publicação na forma de livro em 1996. Moura et al. (2014) introduzem a ideia que dá origem ao presente artigo, fazendo a representação de vários conceitos ligados à produção enxuta na forma da linha de balanço, mas sem se concentrar em áreas específicas ou apresentar conteúdos de forma sistematizada.

Slobodzian (2021) analisa a criticalidade da programação de obras não repetitivas, explorando fortemente a apresentação gráfica dos resultados, além de fazer um apanhado histórico do desenvolvimento da programação de obras.

\section{METODOLOGIA}

Para realização do trabalho foram revisados inúmeros trabalhos presentes na literatura, na forma de dissertações de mestrado, trabalhos de conclusão de curso, artigos e material didático para o ensino de Linha de Balanço e Construção Enxuta buscando-se inspiração em seus desenhos.

De posse do total de transparências disponíveis, buscou-se uma forma de organizá-las em sequência, na medida do possível, vinculando-as com algum desenvolvimento acadêmico ligado a construção enxuta, disponibilizado nos trabalhos dos congressos do IGLC na área de PCP ou em teorizações sobre os princípios que norteiam esta filosofia. Para tal fez-se uso das listagens, dos questionários e das definições de termos encontradas em Rocha et al. (2009), como uma fonte ampla de conceitos a serem ilustrados.

\section{UMA COLEÇÃO DE GRÁFICOS PARA O ENTENDIMENTO DO CONCEITO DE FLUXOS A PARTIR DA LINHA DE BALANÇO}

Os fluxos podem ser definidos como parte integrante do sistema de produção enxuta formado por 3 grandes áreas gerenciais: ações relativas à definição e entrega de valor para clientes (valor), os trabalhos necessários à transformação de inputs em produtos ou 
serviços (transformação) e à movimentação de materiais, de informações e demais insumos, incluindo o próprio produto/serviço em elaboração ao longo do tempo e do espaço (fluxo), conforme Koskela (2000).

A Figura 1 mostra um dos primeiros conceitos associados com a linha de balanço, sob o ponto de vista da transformação: esta é uma técnica que permite identificar quem vai está fazendo o que, onde e quando. Como será visto a seguir, a linha de balanço tem potencialidades para representar o fenômeno produtivo de forma muito mais ampla. A Figura 2 introduz inicialmente o ritmo de produção a modelar na programação de obras.

A Figura 3 introduz ideias ainda ligadas à transformação. Existe um bloco de atividades colocadas junto ao eixo do $\mathrm{x}$ e um ritmo de conclusão de repetições deste primeiro bloco, o que leva a duração final da obra. Mais do que isto, uma parte do trabalho situado ao final da chamada unidade de base (situada junto ao eixo x) é escolhida para representar um fluxo de trabalho que se adequa a um ritmo, o que posteriormente vai ser associado aos tempos "takt” (TOMMELEIN, 2020).

Figuras 1 a 3 - Desenhos esquemáticos para visualização de conceitos lean a partir da linha de balanço - uma introdução
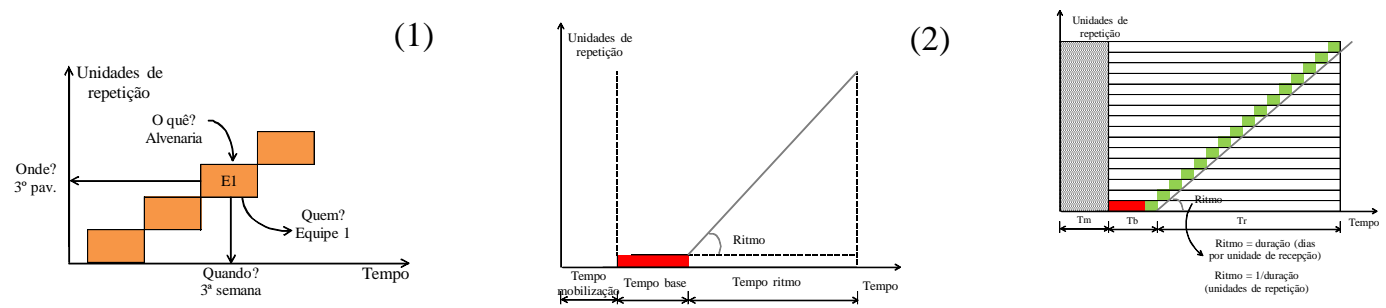

Fonte: Autores (2021)

Tem-se assim, conforme a Figura 4, um fluxo de processos para terminar uma unidade de repetição e um fluxo de operações para planejar a execução desta última atividade ao longo da duração da obra. A Figura 5 apresenta o fluxo de recursos que permeia toda a execução das obras no canteiro, ou em outras palavras, o fluxo de esforço produtivo. Estes recursos (operários, equipes e equipamentos) vão percorrendo o canteiro para concretizar suas responsabilidades. O fluxo dos recursos é tal que pode ser associado a objetivos estratégicos ao longo e ao final da conclusão dos trabalhos, aqui ilustrados como objetivos de tempo, custo e qualidade.

Figuras 4 a 6 - Desenhos esquemáticos para visualização de conceitos lean a partir da linha de balanço - uma introdução

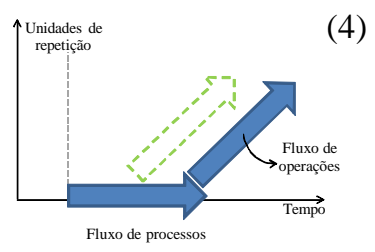

(4)

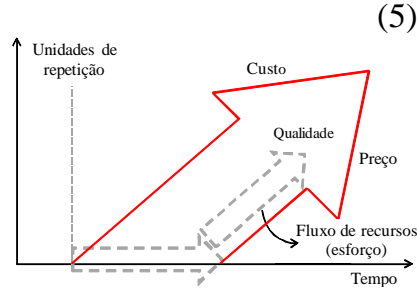

(5)

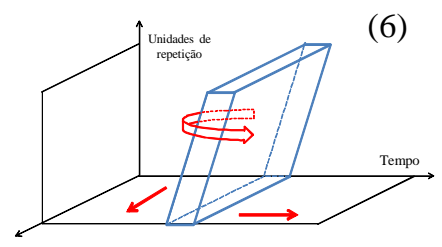

Fonte: Autores (2021)

A Figura 6 associa o objeto em construção, na forma de um prisma, com os diversos fluxos que vão precisar ser planejados e coordenados. Tem-se um fluxo junto às fundações (subsolo e paredes de contenção), um fluxo junto ao térreo (eixo dos x), um fluxo de trabalho ao longo de um mesmo andar, um fluxo de andar para andar e finalmente movimentos ao longo das faces do prisma, emulando os trabalhos relativos à fachada. Neste ponto, apresenta-se, a guisa de exemplo, o argumento apresentado no trabalho de Friblick et al. (2009) sobre a execução de fachadas. Parte da tecnologia desenvolvida por estes está ligada a colocação dos painéis de fachada na obra, em 
termos de fluxos de deslocamento horizontais e verticais, desde a chegada do material em obra.

A Figura 7 toma emprestada uma noção da psicologia positiva para mostrar que o conceito de fluxo é de larga difusão na interpretação das ações humanas (SNYDER; LOPEZ, 2009). Segundo a psicologia positiva, um indivíduo está em fluxo (em flow) quando percorre um caminho ao longo do tempo onde suas capacidades como ser humano e como trabalhador estão de acordo com as demandas que sobre ele são exercidas.

A Figura 8 prepara o caminho para o entendimento de uma analogia frequente na utilização da linha de balanço com os conceitos de construção enxuta, mormente em língua espanhola. A linha de balanço é um "trem de atividades" que atravessa o canteiro de obras. As várias atividades em sequência são como uma locomotiva e seus vagões a serem puxados (ou empurrados) ao longo da obra. A seta inclinada por trás da figura indica o caminho geral do esforço despendido pelas várias atividades em sequência.

A Figura 9 coloca de maneira mais abrangente a representação final de uma linha de balanço. Todas as atividades estão em um fluxo ideal, com ritmos iguais para todas elas, permitindo a existência de uma folga de tempo concentrada no prazo final do prazo contratual. Este gráfico permite a discussão comparativa de Produção Enxuta com os preceitos da Teoria das Restrições, como ilustrado em Shen e Chua (2008).

Figuras 7 a 9 - Esquemas para visualização de conceitos lean a partir da linha de balanço

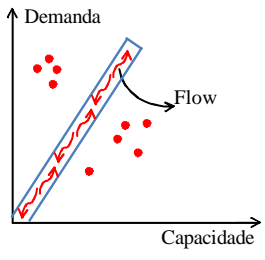

(7)

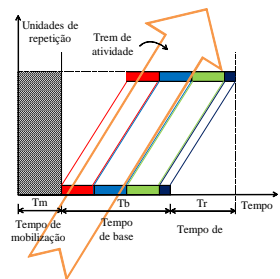

(8)

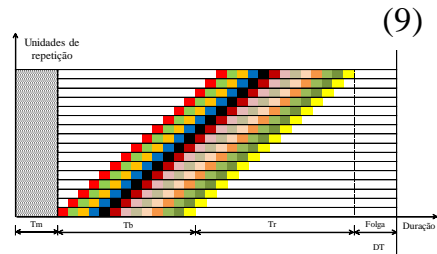

Fonte: (7) a (9) Autores (2021)

As Figuras 10, 11, 12, 13 e 14 mostram gráficos retirados de Pigott (1974) ilustrando dados estatísticos para a realidade irlandesa naquela década. Estes permitem a estimativa do tempo de mobilização, do tempo de base e do tempo de ritmo.

Figuras 10 a 15 - Esquemas para visualização de conceitos lean a partir da linha de balanço
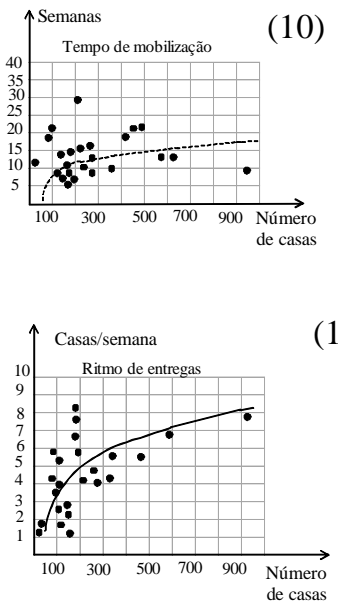

(11)
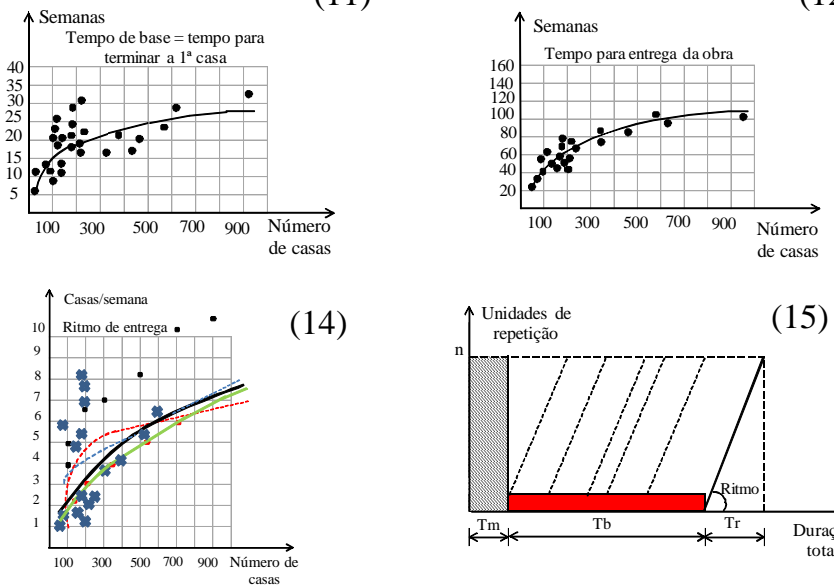

(14)

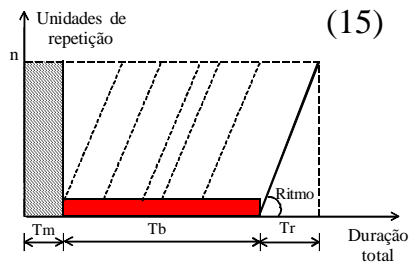

Fonte: (10) a (14) Pigott (1974) e (15) Autores (2021)

A Figura 14 mostra em vermelho uma coerência maior para o ritmo de entrega das unidades do que na Figura 13, já que para cada obra são utilizados os dados das figuras 10 a 12. Estes ritmos elevados permitem a entrega da obra em prazos razoáveis (2 a 3 
anos). A coerência entre os gráficos é buscada a partir da formulação ilustrada na Figura 15 , onde a duração total do contrato é o somatório dos tempos de mobilização, tempo de base e tempo de ritmo. Todas as variáveis podem ser alteradas, mas deve haver coerência entre elas e a duração total da obra.

As Figuras 16 e 17 ilustram o aspecto intuitivo da programação por linha de balanço, e a consideração de ritmo como algo já presente na realidade dos canteiros, mesmo na ausência de aplicações formais desta técnica de programação. A Figura 16 mostra um cronograma de obras clássico, de cima para baixo, com as atividades predecessoras abrindo espaço para que as atividades sucessoras possam ser iniciadas. É como se houvesse um cascateamento de espaços a serem trabalhados, tipicamente a estrutura avançando e abrindo caminho para a execução da alvenaria alguns andares abaixo.

Figuras 16 a 18 - Esquemas para visualização de conceitos lean a partir da linha de balanço
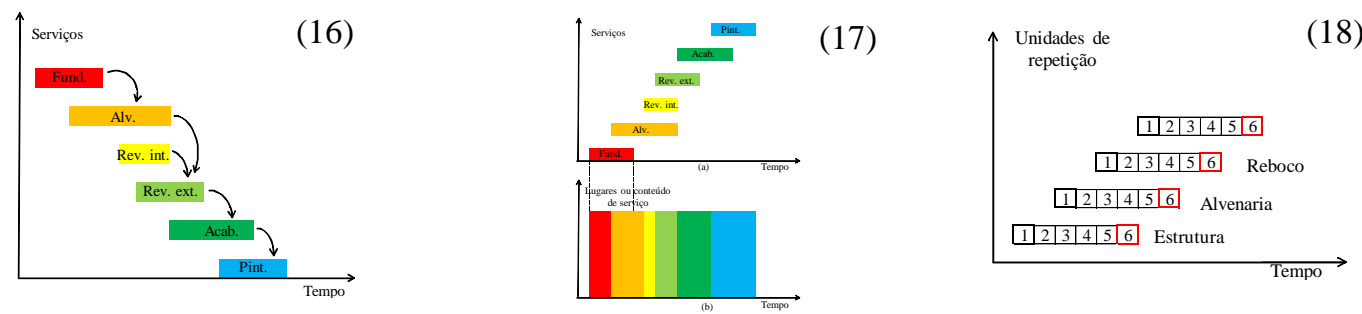

Fonte: (16) a (18) Autores (2021)

Em particular a Figura 17 apresenta um cronograma de barras pouco usual, com as atividades colocadas de baixo para cima. A mensagem é que a linha de balanço consiste em um cronograma de barras, as quais são colocadas de maneira inclinada, segundo o ritmo de trabalho aludido anteriormente. $\mathrm{O}$ fato a notar é que no cronograma de barras (de cima para baixo ou de baixo para cima) se perde um eixo do gráfico (eixo y), onde poderiam ser colocadas informações, no exercício da transparência, para melhorar o entendimento da programação de obra. A Figura 18 melhora o cronograma de obras, dividindo cada barra pelas unidades em sequência que vão ser atacadas dentro de cada serviço.

Por outro lado, a Figura 19 indica uma possibilidade que pode ocorrer em termos práticos e que dificulta a proposta de colocação das unidades de repetição ao longo do eixo dos y. É o caso do seguinte exemplo: a partir da estrutura, todas as atividades em cada unidade de repetição (por exemplo, os andares de um edifício) podem ser feitas em paralelo, como indicado, com fatores de lead/lag (atraso/adiantamento) entre elas. Mesmo assim, aparece uma imagem de fluxo de atividades e de recursos atravessando o canteiro.

Figuras 19 a 21 - Desenhos complementares para visualização de conceitos lean a partir da representação em linha de balanço

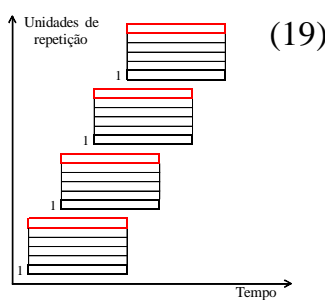

(20)

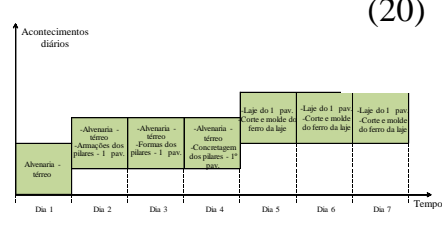

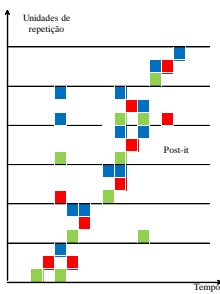

Fonte: (19) a (21) Autores (2021)

As Figuras 20 e 21 dão mais um testemunho de que já é praticado um fluxo de forma intuitiva em obra, ainda que não ordenado. Se as folhas de um diário de obra forem colocadas em um quadro, fazendo referência às unidades de repetição que são majoritariamente descritas neste texto vai ser possível criar a imagem de um fluxo de 
atividades atravessando o canteiro. Da mesma forma, um quadro de "post it", como discutido em Ribeiro et al. (2017), usado no planejamento de fase, também vai dá esta impressão de fluxo ao longo do canteiro, como ilustrado na Figura 20.

A figura 22 faz a união entre os conceitos de Transformação e Fluxo da Teoria. Ao longo do eixo dos x são estabelecidos porções de trabalho a serem executadas segundo preceitos técnicos e gerenciais próprios da transformação. Ao longo do eixo dos y, as unidades de repetição são colocadas, ou modernamente, os locais de execução. A figura é tal que não impõe dimensões para os ciclos ao longo do tempo e do espaço: o importante é a definição destes ciclos nas 2 direções.

A Figura 23 reforça o conceito de que o fundamental, para início das implementações da Construção Enxuta, é a definição dos ciclos nas duas direções. Quaisquer que sejam estes ciclos, isto permitirá o delineamento de um fluxo de atividades a percorrer os canteiros de obras. Em termos ideais, ao longo da evolução no uso da construção enxuta vão se tornando menores os ciclos de tempo e de espaço.

A Figura 24 sugere onde se objetiva chegar em termos de transparência e simplicidade. Um primeiro passo seria acompanhar poucos ciclos de espaço que se repetem de unidade em unidade, seguindo um certo ritmo único, com tamanhos de ciclos de tempo relativamente semelhantes.

Figuras 22 a 24 - Desenhos complementares para visualização de conceitos lean a partir da representação em linha de balanço

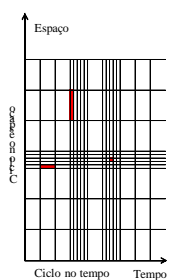

(22)

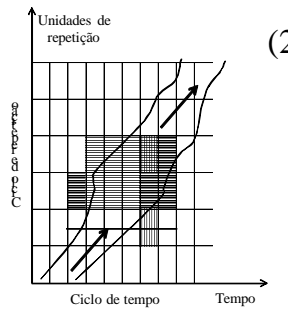

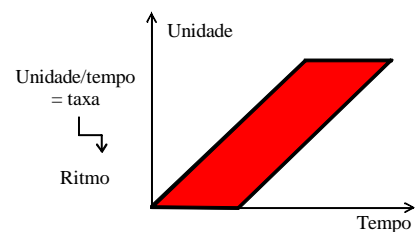

Fonte: (22) a (24) Autores (2021)

As Figuras 25 e 26 ilustram uma dificuldade típica na identificação deste fluxo ao atravessar o canteiro, agora no caso de uma atividade específica. Claramente a Figura 26 mostra um fluxo ordenado, que é apresentado na Figura 25, no entanto, por um fluxo aleatório. Ocorre que a ordenação do eixo dos y não respeitou a exata sequência topológica com que a obra seria executada. Havia fluxo, mas a representação deste fluxo não era perceptível.

Figuras 25 a 27 - Desenhos complementares para visualização de conceitos lean a partir da representação em linha de balanço
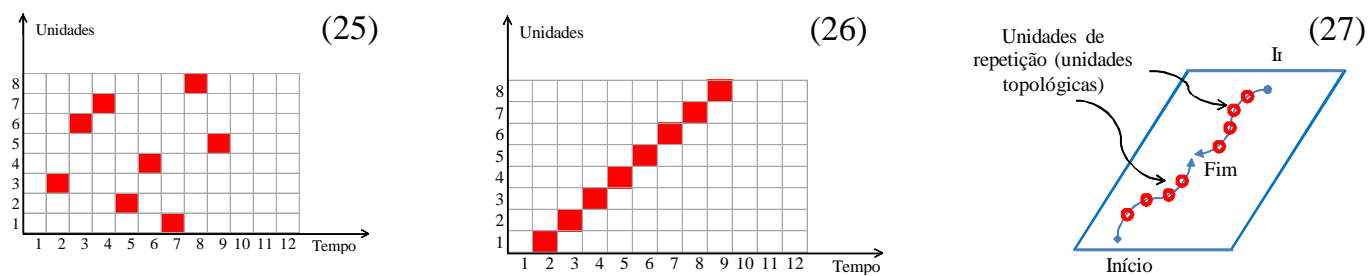

Fonte: (25) a (27) Autores (2021).

Roesch (1984) introduz a noção de topologia nos canteiros, através das Figuras 27, 28 e 29. Por topologia entendem-se os locais a serem trabalhados no canteiro e a sua sequência. Modernamente, isto veio a dar origem ao sistema de planejamento LBMS, fortemente ancorado nos conceitos tradicionais da linha de balanço, como sintetizado por Seppännen (2014), o principal autor da área. Na Figura 27 ilustra-se o caso de 2 
frentes de trabalho que dão entrada no canteiro em lados opostos e vêm a se encontrar na região central. A linha de balanço (ou a LBMS) para esta obra adquire a forma de uma flecha na Figura 28, a melhor imagem de um fluxo de esforço produtivo.

$\mathrm{Na}$ Figura 29 Roesch sugere vários planos de ataque para o canteiro, incluindo a possibilidade de múltiplas equipes para o mesmo serviço, que dão entrada no canteiro por várias frentes. O sequenciamento dos locais de execução pode não ser trivial, implicando dificuldades na observação de um fluxo, mesmo quando ele existe. A Figura 30 mostra a sequência de operações ligadas à alvenaria portante, as lajes de entrepiso e a cobertura do prédio. As alvenarias são executadas alternadamente em 2 blocos em sequência, para haver tempo de execução da laje de entrepiso no bloco anterior. Isto faz com que se tenha um atraso para liberar a execução das coberturas, na medida em que esforço produtivo foi alocado lateralmente. Este, com outra tecnologia de construção, poderia ter sido alocado para liberar a execução das coberturas o mais rápido possível.

Figuras 28 a 30 - Desenhos complementares para visualização de conceitos lean a partir da representação em linha de balanço

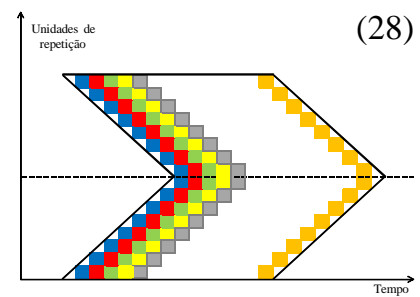

(28)
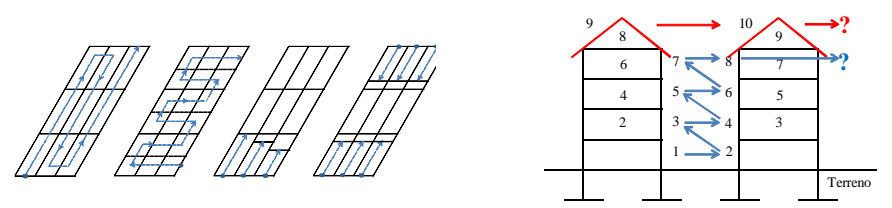

Fonte: (28) e (30) Autores (2021); (29) Roesch (1984)

\section{CONSIDERAÇÕES FINAIS}

Os gráficos apresentados dão testemunho da capacidade da linha de balanço representar vários conceitos apresentados sobre produção enxuta, especificamente nos congressos do IGLC. Segundo o que foi apresentado no presente artigo, não foram encontradas dificuldades para ilustrar os conceitos de ciclo e de fluxo, em particular o último, através do uso da técnica de programação da linha de balanço. Da mesma forma, no relatório original de Pinheiro (2009), resumido em sua parte inicial na presente contribuição, não foram encontradas dificuldades para representar 20 de um total de 56 conceitos de produção enxuta escolhidos.

A linha de trabalho adotada aqui visa organizar de maneira mais abrangente os cerca de 328 conceitos ilustrados em Rocha (2009). A contribuição aqui apresentada explora os conceitos de fluxos no canteiro de obras. Trabalhos futuros poderão abranger um espectro maior de ações, técnicas e conceitos imanentes a esta filosofia gerencial permitindo o delineamento de material instrucional para ensino de programação de obra alicerçado na chamada Lean Construction, conforme propiciado pela prolífica literatura de ambas as áreas.

\section{REFERÊNCIAS}

BALLARD, G.; HOWELL, G. A. Implementing lean construction: stabilizing work flow. Lean Construction, editor L. F. Alarcon, 1st (1993), 2nd (1994), 3rd (1995) International Group for Lean Construction Conferences, Balkema Publishing, 1996.

FORMOSO, C. T. A Knowledge based Framework for Planning House Building Projects.1991. Thesis, University of Salford, Salford, UK, 1991.

FRIBLICK, F.; TOMMELEIN, I. D.; MUELLER, E.; FALK, J. H. Development of an integrated façade system to improve the high-rise building process. In: 17th 
INTERNATIONAL GROUP FOR LEAN CONSTRUCTION CONFERENCE, Taipei, Taiwan, p. 359-370, 2009.

KOSKELA, L. An exploration towards a production theory and its application to construction. 2000. 296p. Thesis, Doctor of Philosophy, VTT Building Technology, Espoo, Finland, 2000.

LUMSDEN, P. The Line of Balance Method. Oxford: Pergamon Press, 1968, 72 p.

MADERS, B. T. Técnica de Programação e Controle de Construções Repetitivas Linha de Balanço: Estudo de Caso em um Conjunto Habitacional. Dissertação (Mestrado em Engenharia Civil), Universidade Federal do Rio Grande do Sul, Porto Alegre, 1987.

MOURA, R. S. L. M.; MONTEIRO, J. M. F.; HEINECK, L. F. M. Line of Balance - is it a synthesis of lean production principles as applied to site programming of Works? In: 22nd INTERNATIONAL GROUP FOR LEAN CONSTRUCTION CONFERENCE, Oslo, Norway, p. 703-714, 2014.

PIGOTT, P. T. A Productivity Study of House Building. 2nd. Impression. Dublin, Ireland, An Foras Forbartha - The National Institute for Physical Planning and Construction Research, 1974.

PINHEIRO, M. B. Considerações gráficas sobre a ligação entre a Linha de Balanço e o Sistema Toyota de Produção. 2009. 57p. Trabalho de Conclusão de Curso, Departamento de Estruturas e Edificações, Universidade Federal do Ceará, Fortaleza, 2009.

RIBEIRO, F. S.; COSTA, D. B.; MAGALHAES, P. A. Phase schedule implementarion and the impact for subcontractors. In: 25th INTERNATIONAL GROUP FOR LEAN CONSTRUCTION CONFERENCE, Heraklion, Greece, p. 687-694, 2017.

ROCHA, F. E. M.; PEREIRA, P. E.; LEITE, M. O. Introdução aos Conceitos Lean visão geral do assunto. Coleção Edificar Lean, v. 1, Fortaleza, Ceará, 2009.

ROESCH, W. T. The Transformation Method - a successful technique for projects with overlapping activities (fast track). In: 4th W65 INTERNATIONAL CONGRESS ON ORGANIZATION AND MANAGEMENT OF CONSTRUCTION, Waterloo, Ontário, Canada, p.603-611, 1984.

SEPPÄNEN, O. A comparison of takt time and LBMS planning methods. In: 22nd INTERNATIONAL GROUP FOR LEAN CONSTRUCTION CONFERENCE, Oslo, Norway, p. 727-738, 2014.

SHEN, L.; CHUA, D. K. H. An investigation of critical chain and lean project scheduling. In: 16th INTERNATIONAL GROUP FOR LEAN CONSTRUCTION CONFERENCE, Manchester, UK, p. 679-690, 2008.

SNYDER, C. R.; LOPEZ, S. J. Psicologia Positiva - uma Abordagem Científica e Pratica das Qualidades Humanas. Porto Alegre: Artmed, 2009.

SLOBODZIAN, J. Planejamento baseado em locais em obras não repetitivas com ênfase na criticalidade. 2001. Dissertação de Mestrado, Faculdade de Engenharia Civil, Arquitetura e Urbanismo, Universidade Estadual de Campinas, São Paulo, 2001.

TOMMELEIN, I. D. Takting the parade of trades: use of capacity buffers to gain work reliability. In: 28TH INTERNATIONAL GROUP FOR LEAN CONSTRUCTION CONFERENCE, Berkeley, California, USA, p. 421-432, 2020. 